\title{
Leitthema
}

Chirurg 2016 · 87:13-19

DOI 10.1007/s00104-015-0115-8

Online publiziert: 21. Dezember 2015

(c) Die Autor(en) 2015. Dieser Artikel ist auf

Springerlink.com mit Open Access verfügbar

CrossMark
O. van Ruler - M.A. Boermeester

Department of Surgery, Academic Medical Center, Amsterdam, Niederlande

\section{Die chirurgische Therapie der sekundären Peritonitis}

\section{Ein weiter andauerndes Problem}

\begin{abstract}
Die Therapie der sekundären Peritonitis oder auch abdominellen Sepsis wird weiterhin kontrovers diskutiert. Morbiditäts- und Mortalitätsraten sanken in der zurückliegenden Dekade nur geringfügig, obwohl die medizinische Versorgung in den westlichen Industrieländern insgesamt weiter deutlich verbessert wurde. Ursachen und Schweregrad der sekundären Peritonitis sowie auch der zeitliche Ablauf vom klinischen Ausbruch bis zum Behandlungsbeginn können sich von Patient zu Patient sehr heterogen gestalten. In dieser Übersicht werden die gegenwärtig wichtigsten Aspekte, speziell der chirurgischen Therapie bei sekundärer Peritonitis, zusammengefasst.
\end{abstract}

\section{Definition der sekundären Peritonitis}

Die sekundäre Peritonitis ist definiert als eine akute, durch Verlust der Integrität des Gastrointestinaltraktes oder anderer viszeraler Organe hervorgerufene Infektion des Peritoneums. Ursächlich verantwortlich für eine sekundäre Peritonitis sind spontane Perforationen viszeraler Organe (z. B. Kolondivertikulitis, Appendizitis und Cholezystitis), traumatisch verursachte Organperforationen oder auch

Übersetzt von Prof. Dr. M. Gasser, Klinik für Allgemein-, Viszeral-, Gefäß- und Kinderchirurgie Universitätsklinikum Würzburg, E-Mail: Gasser_M@ukw.de,Würzburg, 27.09.15

Die englische Version dieses Beitrags ist unter doi:10.1007/s00104-015-0121-x zu finden iatrogene Ursachen (z. B. Anastomoseninsuffizienz; [1]). Schwere Ausprägungen einer sekundären Peritonitis oder abdominellen Sepsis sind auch heute noch, bedingt durch ein Multiorganversagen („multiple organ failure“, MOF), oftmals hervorgerufen durch einen septischen Schock, durch hohe Morbiditäts- und Mortalitätsraten gekennzeichnet. Berichtete Mortalitätsraten sanken in den zurückliegenden Dekaden nur geringfügig und liegen weiterhin zwischen 20 und $60 \%$ insgesamt. Die Morbiditätsraten sind mit $50 \%$ unverändert hoch und zumeist verbunden mit längerem Aufenthalt auf der Intensivstation und längerer Hospitationsdauer [2, 4]. Obwohl die genaue Inzidenz der abdominellen Sepsis nicht bekannt ist, wird sie doch als zweithäufigste Ursache einer Sepsis angenommen [3].

\section{Initiale Therapie}

\section{Operative Therapie}

Entscheidend bei der Therapie einer sekundären Peritonitis ist die unverzügliche Sanierung des Infektionsherdes, begleitet von kardiopulmonalen Unterstützungsmaßnahmen und einer antimikrobiellen Therapie [4]. Die Behandlung zielt also auf eine Kontrolle des septischen Infektionsherdes und die Prävention einer sich ausbreitenden Infektion ab. Die unverzügliche Infektionskontrolle wird beispielsweise durch Resektion eines perforierten viszeralen Hohlorgans, abhängig von Ätiologie und Lokalisation sowie Komorbiditäten des Patienten, mit Wiederherstellung der Darmkontinuität erreicht $[1,5,6]$.
Häufig werden abdominelle Spülungen mit einfacher Kochsalzlösung mit oder ohne hinzugefügte Antibiotika sowie auch mit antiseptischen Lösungen vorgenommen. Dabei zeigen keine dieser letztgenannten Lösungen einen positiven Effekt auf das Überleben einer sekundären Peritonitis [5]. Im Gegenteil ist davon auszugehen, dass bei abdominellen Spülungen die mesothelialen Zellen, die eine wichtige Rolle in der Immunantwort des Patienten spielen, mit entfernt werden [7]. Ein reiner Verdünnungseffekt der Infekterreger in der Bauchhöhle sollte, da darüber hinaus potenziell schädigend, bei allen abdominellen Spülungen eher vermieden werden.

\section{Kardiopulmonale Unterstützung}

Eine sekundäre Peritonitis mit möglicher Folge einer generalisierten Sepsis erfordert nach den Empfehlungen der Surviving Sepsis Campaign zumeist eine adäquate kardiopulmonale Unterstützung. Allein durch eine inadäquate Gewebeperfusion kann sich im Rahmen einer Sepsis ein Multiorganversagen ausbilden. Kardiopulmonal unterstützende Maßnahmen umfassen somit alle Möglichkeiten zur Aufrechterhaltung oder Verbesserung der Organperfusion und -oxygenierung. Adäquate Maßnahmen, die innerhalb von $6 \mathrm{~h}$ nach Ausbruch einer Sepsis eingeleitet werden, steigern damit das Überleben betroffener Patienten [8].

\section{Antimikrobielle Therapie}

Von entscheidender Bedeutung bei einer Sepsis auf dem Boden einer Peritonitis ist die frühzeitige Einleitung 
einer empirischen Antibiotikatherapie. Jede Verzögerung in der initialen Antibiotikagabe, wie anhand von jeweils 30-minütigen Zeitabständen aufgezeigt, steigerte das Risiko an einer sekundären Peritonitis zu versterben mit einer Odds Ratio (OR) von 1,021 (95\%-Konfidenzintervall[CI]: 1,003-1,038; [9]). Der Vorteil einer frühen und adäquaten Antibiotikatherapie wurde durch die Reduktion der Mortalität der Patienten mit Bakteriämie auf der Intensivstation eindrücklich demonstriert (Risikoreduzierung $33 \%$; [10]). Eine CochraneAnalyse zu diesem Thema beschrieb zurückliegend vergleichbare Effekte der gegenwärtig entwickelten therapeutischen Regime [11]. Wiederum bedarf es angepasster Vorgehensweisen je nach erwartetem Keimspektrum; entsprechend sollte die Therapie beim Vorliegen der mikrobiellen Befunde und dem Antibiogramm ggf. umgestellt werden [12].

\section{Antifungale Therapie}

Ein erheblicher Anteil an Patienten mit Peritonitis auf den Intensivstationen weist Kolonisationen mit Hefen und verschiedenen Pilzstämmen, zumeist Candida-Spezies, auf [13]. Eine Metaanalyse zeigte dazu ein vermindertes Risiko einer Infektion mit Hefen, wenn sowohl eine antifungale Einzelprophylaxe als auch eine selektive Darmdekontamination durchgeführt wurden (OR 0,54 [95\%-CI: $0,39-0,75$; „number needed to treat" (NNT) 20] und 0,29 [95\%-CI: 0,18-0,45; NNT 18]). Ebenso reduzierte eine Prophylaxe das Risiko an einer Infektion mit Hefen zu versterben, unabhängig davon, ob eine antifungale Prophylaxe oder aber eine selektive Darmdekontamination erfolgten (kombiniert OR: 0,23; 95\%-CI: 0,09-0,6 NNT 41; [14]).

> Angesichts der zunehmenden Anzahl an Infektionen mit Hefen wird entsprechend eine Prophylaxe bei Hochrisikopatienten empfohlen.

Bekannte Risikofaktoren stellen Operationen, eine nosokomiale Peritonitis, Perforationen im oberen Gastrointestinaltrakt, eine Immundefizienz, Langzeitantibiotikatherapie, ein akutes Nierenversagen und ein zentralvenöser Zugang dar [15].

\section{Vorgehen nach initialer Laparotomie}

\section{Chirurgische Strategie}

Abhängig davon, ob eine Peritonitis in milder oder schwerer Ausprägungsform vorliegt, werden unterschiedliche chirurgische Strategien verfolgt. Bislang findet sich in der Literatur allerdings kein klarer Konsens zur Definition einer schweren Peritonitis, ebenso nicht zur Frage, welches klinische Scoring benutzt werden sollte und welche Schwellenwerte adäquat die Schwere einer Peritonitis unterscheiden. Der APACHE (Acute Physiology And Chronic Health Evaluation) -II-Score wird dazu am meisten akzeptiert, mit Werten $\leq 10$ für eine milde Peritonitis und $>10$ für schwere Ausprägungsformen [4]. Verschlechtert sich das klinische Bild eines Patienten bzw. bleibt eine Befundbesserung während der ersten postoperativen Phase nach initialer Laparotomie aus, wird eine Relaparotomie unter On-demand-Strategie notwendig [4]. Schwere Formen einer Peritonitis beinhalteten in der Vergangenheit ein aggressiveres chirurgisches Vorgehen mit radikalem intraabdominellem Débridement und eine Behandlung als „offenes Abdomen“ unter geplanter Relaparotomie. Sowohl das radikale Débridement als auch das Vorgehen unter offenem Abdomen sind inzwischen nicht mehr angewandte Strategien, da sich darunter höhere Morbiditäts- und Mortalitätsraten zeigten [16].

\section{》) Die Damage-control- Chirurgie gewinnt fälschlicherweise zunehmend an Bedeutung}

Vor dem Hintergrund der beobachteten negativen Ergebnisse unter der Strategie des geplant offenen Abdomens gewann ein Prinzip aus der Traumatologie, die Damage-control-Chirurgie, in den zurückliegenden Jahren für die Behandlung der Peritonitis zunehmend an Bedeutung. Bei diesem gewählten
Vorgehen wird der Infektionsherd chirurgisch umgehend versorgt und das Abdomen unter Einlage eines (resorbierbaren) Netzes in Inlay-Position temporär verschlossen sowie ein Vakuumverband als Wundsystem darüber angelegt. Der definitive Bauchdeckenverschluss sollte, sobald möglich, verzögert erfolgen, wird jedoch nicht in allen Fällen tatsächlich erreicht. Diese Strategie beinhaltet mehrfache chirurgische Eingriffe über Tage bis Wochen. Bislang sind für ein solches Vorgehen einer Damage-control-Chirurgie wenig Langzeitergebnisse bekannt, da nur kleinere retrospektive Fallserien zurückliegend veröffentlicht wurden [17].

Eine Strategie des offenen Abdomens erlaubt einen direkten Zugang für eine Relaparotomie und verhindert ein abdominelles Kompartmentsyndrom. Dem stehen jedoch gefürchtete Komplikationen bei offenem Abdomen gegenüber, wie die einer Anastomoseninsuffizienz, der Ausbildung enteraler Fisteln in das offene Abdomen (10-20\%), eines Ileus, exzessiver Flüssigkeitsverluste, einer Blutung aus dem offenen Abdomen, sekundärer Infektionen (über $80 \%$ ), verbleibender Fasziendehiszenz, die einer ventralen Bauchwandhernie sowie einer erhöhten Mortalitätsrate. Zudem wird an vielfach unterschiedlichen technischen Vorgehensweisen festgehalten. Damit ist die Evidenz gering und überprüfte Empfehlungen können nur schwerlich ausgesprochen werden [18-20]. Unter diesen Gesichtspunkten mit den substanziell negativen Folgen eines offenen Abdomens empfehlen wir, soweit möglich, das Abdomen zu verschließen und sich nicht für eine Strategie eines geplant offenen Abdomens zu entscheiden. Sollte das Abdomen aufgrund eines generalisierten viszeralen Ödems nicht zu verschließen sein, stehen verschiedene Möglichkeiten eines temporären Verschlusses zur Verfügung [20]. Ein sekundärer Faszienverschluss ist darunter zwar eher selten möglich und die Rate an auftretenden enteralen Fisteln bleibt signifikant erhöht. Zudem bedarf es für einen oberflächlichen Bauchdeckenverschluss zumeist mehrfacher Wechsel des Auflagensystems und operativer Revisionen (• Abb. 1; [20]). 
Chirurg 2016·87:13-19 DOI 10.1007/s00104-015-0115-8

○ Die Autor(en) 2015. Dieser Artikel ist auf Springerlink.com mit Open Access verfügbar

\section{O. van Ruler · M.A. Boermeester}

\section{Die chirurgische Therapie der sekundären Peritonitis. Ein weiter andauerndes Problem}

\section{Zusammenfassung}

Hintergrund. Die sekundäre Peritonitis ist bis heute durch unverändert hohe Morbiditäts- und Mortalitätsraten gekennzeichnet und bleibt auch in Zeiten moderner medizinischer Versorgung therapeutisch eine Herausforderung. Die chirurgische Intervention zur Fokuskontrolle bleibt neben einer adäquaten antimikrobiellen Therapie und, wenn notwendig, intensivmedizinischen Maßnahmen zentrales Element in der Therapie der sekundären Peritonitis. Die Relaparotomie „on demand“ (ROD) nach initialer chirurgischer Versorgung stellt sich nach den Ergebnissen einer randomisierten klinischen Studie als das bevorzugte therapeutische Vorgehen unabhängig von der Schwere der Peritonitis dar.

Ergebnisse und Diskussion. Für ein effektives und sicheres Vorgehen unter ROD bedarf es eines intensiven Monitorings des Patienten mit verschiedenen diagnostischen Testmethoden und klaren Entscheidungsprozessen für eine Relaparotomie, beides ist über $24 \mathrm{~h}$ am Tag vorzuhalten. Mangelndes Wissen um eine frühzeitige und adäquate Patientenselektion sowie fehlender Einsatz durchaus einfacher diagnostischer Verfahren scheinen die Umsetzung des Konzeptes und Entscheidung zur ROD im Einzelfall eher zu erschweren. Dabei zeigt sich die prädiktive Genauigkeit bei Anwendung eines überprüften Entscheidungsprogramms für eine Relaparotomie als angemessen zur Vorhersage einer sich ausbildenden Peritonitis und notwendigen Patientenselektion für eine sich ableitende computertomographische (CT-)Diagnostik. Die Wertigkeit einer CTDiagnostik in der frühen postoperativen
Phase bleibt bislang unklar. Zukünftige Studien und innovative Technologien sollten die Bedeutung einer additiven CT-Diagnostik nach chirurgischer Therapie bei sekundärer Peritonitis aufgreifen. Das beinhaltet aber auch weiter optimierte bettseitige Entscheidungshilfen, um Patienten adäquat in einem multidisziplinären Umfeld für einzelne Interventionen auswählen zu können.

\section{Schlüsselwörter}

Peritonitis · Abdominelle Sepsis - Geplante Relaparotomie - Relaparotomie on demand . Behandlungsstrategien

\section{Surgical treatment of secondary peritonitis. A continuing problem. German version}

\section{Abstract}

Background. Secondary peritonitis remains associated with high mortality and morbidity rates. Treatment of secondary peritonitis is still challenging even in the era of modern medicine. Surgical intervention for source control remains the cornerstone of treatment besides adequate antimicrobial therapy and when necessary intensive medical care measures and resuscitation. A randomized clinical trial showed that relaparotomy on demand (ROD) after initial emergency surgery was the preferred treatment strategy, irrespective of the severity and extent of peritonitis.
Results and discussion. The effective and safe use of ROD requires intensive monitoring of the patient in a setting where diagnostic tests and decision making about relaparotomy are guaranteed round the clock. The lack of knowledge on timely and adequate patient selection, together with the lack of use of easy but reliable monitoring tools seem to hamper full implementation of ROD. The accuracy of the relaparotomy decision tool is reasonable for prediction of the formation of peritonitis and necessary selection of patients for computed tomography (CT). The value of $\mathrm{CT}$ in the early postoperative phase is unclear. Future research and innovative technologies should focus on the additive value of CT after surgical treatment for secondary peritonitis and on the further optimization of bedside prediction tools to enhance adequate patient selection for interventions in a multidisciplinary setting.

\section{Keywords}

Peritonitis · Abdominal sepsis · Planned relaparotomy - On-demand relaparotomy . Treatment strategy
Als potenzielle Alternative steht hierzu die Technik des Bauchdeckenverschlusses mit einem biologischen Netz zur Verfügung. Der frühe Verschluss noch während der initialen Operation oder kurzfristig danach bei restlich noch kontaminierten Wundverhältnissen mit einem biologischen Netz kann eine unmittelbare Lösung bedeuten und therapeutisch die Rate an Fisteln und Narbenhernien reduzieren. Geplante Relaparotomien beinhalten dagegen Revisionseingriffe im Abstand von zwei bis drei Tagen bis die Abdominalhöhle makroskopisch aus infektiologischer Sicht sauber erscheint, weitgehend unabhängig von den klinischen Bedingungen des Patienten [5, 21].

Ein geplantes strategisches Vorgehen basierte in der Vergangenheit auf der Annahme eines Vorteils, eine persistierende Peritonitis oder neue Infektionsherde frühzeitig zu identifizieren und entsprechend zu behandeln. Dagegen erhöhte es die Anzahl unnötiger Relaparotomien [4]. Jüngere Daten zeigen auch für schwere Formen der Peritonitis, dass ein „on demand“ chirurgisches Vorgehen die Therapie der Wahl zu sein scheint [4]. Weitere Ergebnisse weisen zudem auf eine gesteigerte inflammatorische Mediatorantwort und eine erhöhte Rate an Multi- organversagen sowie Mortalität unter dem Vorgehen multipler Relaparotomien hin [22].

In einer eigens durchgeführten randomisierten und kontrollierten Studie (RELAP-Trial) wurde das Vorgehen der geplanten Relaparotomie mit der Relaparotomie „on demand“ (ROD) verglichen [4]. Von insgesamt 232 untersuchten Patienten wurden jeweils 116 Patienten mit moderater bzw. schwerer sekundärer Peritonitis (APACHE-II-Score $>10$ ) in den Arm mit geplanter vs. Ondemand-Laparotomie eingeschlossen. Die Mortalität lag im Vergleich zur Gruppe mit geplanter Relaparotomie mit 

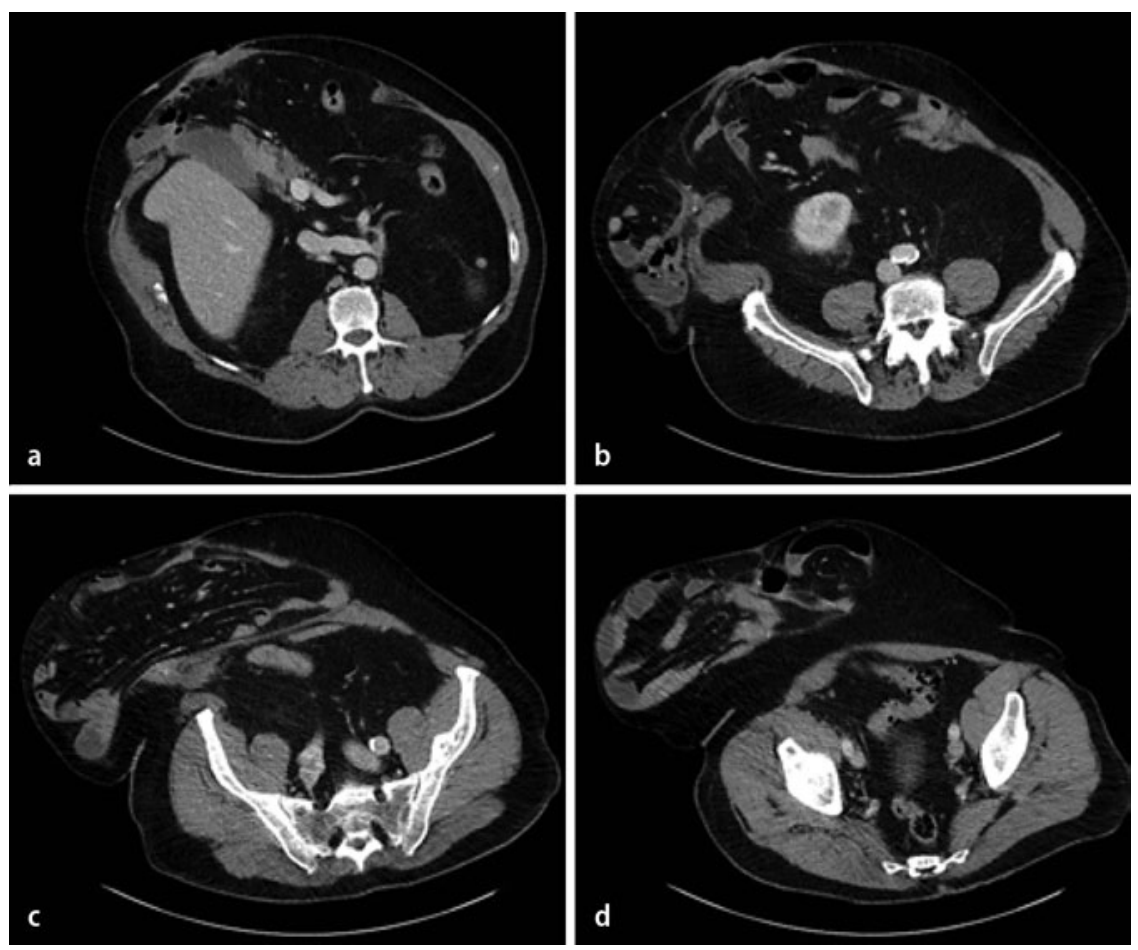

Abb. $1 \Delta$ Beispiel für die verhängnisvollen Folgen eines geplant offenen Abdomens: Fistelbildung in das offene Abdomen mit vorhandenen Resten eines Kunststoffnetzes für den temporären Bauchdeckenverschluss. Dargestellt sind vier Schichten zwischen Ober- und Unterbauch (a-d) mit erkennbarer Dünndarmfistel $(\mathbf{a}, \mathbf{b})$

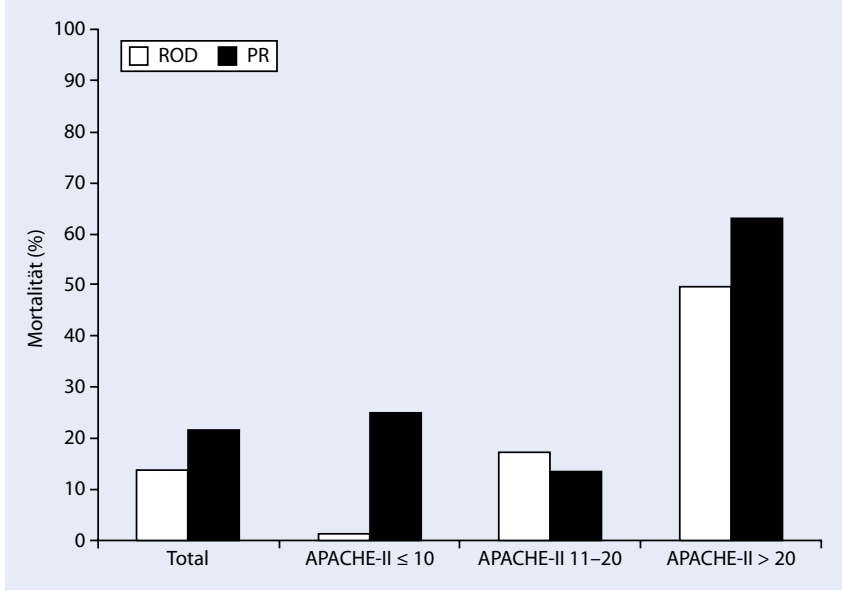

$36 \%$ in der mit ROD leicht niedriger bei $29 \%(p=0,22)$. Auch bei den Patienten mit besonders schwerem Krankheitsbild bei sekundärer Peritonitis (APACHE-IIScore $>20$; - Abb. 2) zeigte sich kein verbessertes Überleben bei jeweils geplant durchgeführter Relaparotomie. Diese Ergebnisse stehen im Widerspruch zu der weitläufigen Annahme, dass insbesondere Patienten mit schwerem Krankheitsbild von einem Vorgehen mit geplanter Relaparotomie profitieren würden. Ein weiteres unbestätigtes Dogma zielt auf
Abb. $2<$ Mortalitätsraten stratifiziert für das Vorgehen einer Relaparotomie on demand (ROD) und einer geplanten Relaparotomie (PR) sowie der Krankheitsschwere bei untersuchten Patienten in der RELAP-Studie

Gesamtverweildauer im Krankenhaus die medizinischen Behandlungskosten. So sanken die Gesamtkosten um durchschnittlich 17.500 EUR pro Patient [23]. Patienten unter On-demand-Strategie wurden weniger häufig einer Relaparotomie unterzogen (113 vs. $233 \mathrm{Re}$ operationen in der Gruppe mit geplanter Relaparotomie) und benötigten in mehr als der Hälfte der Fälle (58\%) keine Relaparotomie. Darüber hinaus lag die Rate an negativen Relaparotomien (ohne persistierende oder neue Infekt-Foci) in der Gruppe mit On-demand-Relaparotomie deutlich niedriger (On-demand-Gruppe $31 \%$ vs. $66 \%$ in der Gruppe mit geplanter Relaparotomie; [4]).

$>$ Negative Relaparotomien sind klar als unnötige Prozeduren mit vermeidbarem Gefährdungspotenzial zu betrachten.

Eine verbesserte Patientenselektion für eine interventionelle computertomographische (CT-)Diagnostik kann somit dazu beitragen, den Anteil an negativen Relaparotomien unter der Ondemand-Strategie weiter zu reduzieren. Ferner können perkutane Drainagen, die interventionell in infizierte Flüssigkeitskollektionen eingelegt werden, mit helfen, die Rate an Relaparotomien zu senken. Trotz der jüngsten klinischen Ergebnisse aus dem Vorgehen einer Ondemand-Relaparotomie in der eigenen Studie, in anderen Veröffentlichungen auch als konventionelle Behandlungsstrategie beschrieben, und der günstigen ökonomischen Aspekte bleibt das Vorgehen mit geplanter Relaparotomie doch das weiterhin eher vorrangig gewählte Verfahren.

Für ein erfolgreiches Vorgehen mit einer Relaparotomie nach klinischem Verlaufsbild (on demand) bedarf es der Einführung einer adäquaten Überwachung des Patienten im Sinne einer sogenannten Damage-control-Strategie. Hierauf wird im Weiteren eingegangen.

\section{Monitoring}

Eine On-demand-Strategie reduzierte zuverlässig durch die sowohl verkürzte Aufenthaltsdauer auf der Intensivstation als auch durch die verkürzte
Bislang gibt es keine klaren Entscheidungshilfen in der Auswahl eines Patienten für eine Relaparotomie. Die 

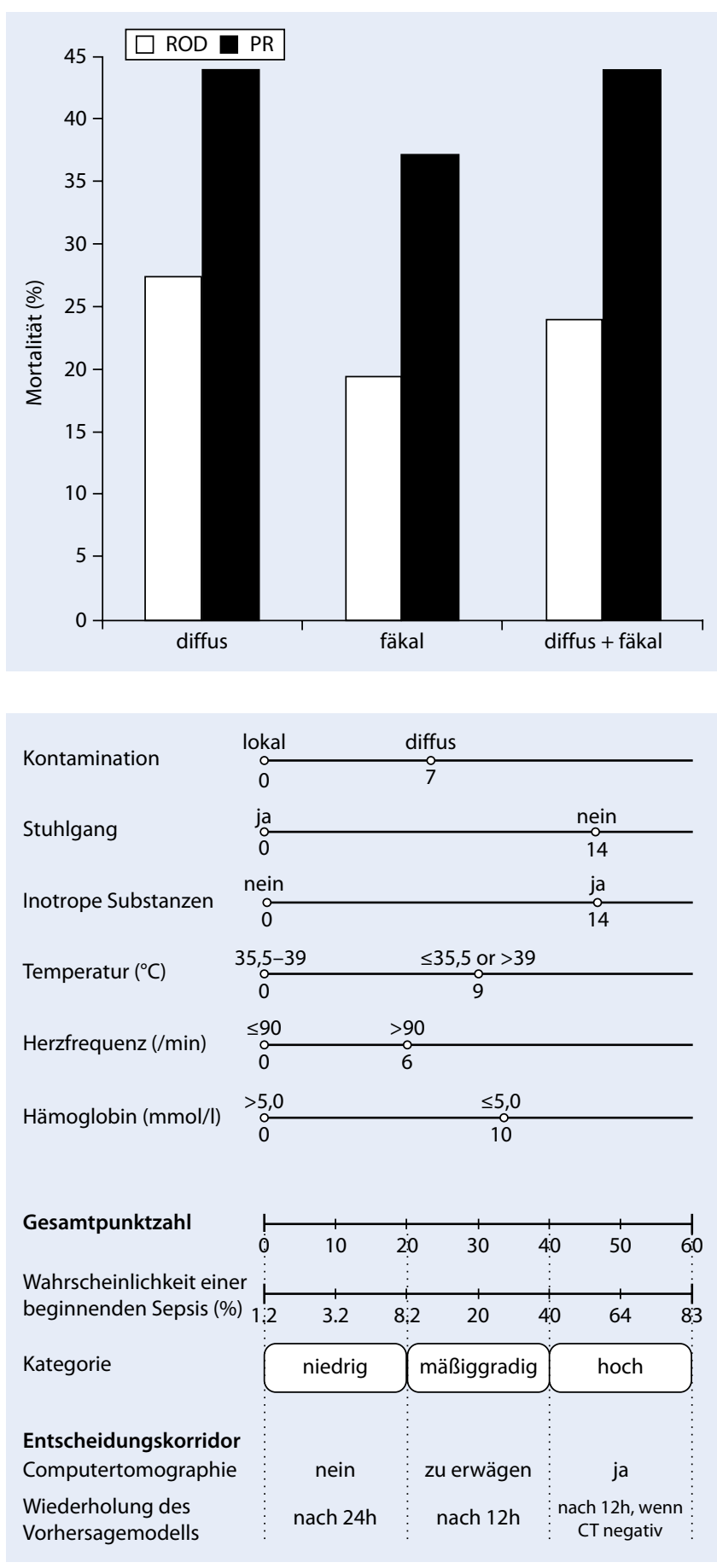

Abb. $3<$ Mortalitätsraten stratifiziert für das Vorgehen einer Relaparotomie on demand (ROD) und einer geplanten Relaparotomie (PR) sowie der Art der abdominellen Kontamination bei untersuchten Patienten in der RELAP-Studie

Abb. $4<$ Beispielhaftes Nomogramm mit einem dargestellten Ergebnis aus dem Entscheidungsprogramm zur Vorhersage einer sich ausbildenden abdominellen Sepsis und Empfehlung zur Überwachung und Durchführung einer bildgebenden Diagnostik. (Adaptiert nach [25])
Indikationsstellung basiert vielmehr auf einer subjektiven Interpretation undefinierter klinischer Variablen. Es existieren auch keine prädiktiven Parameter, die geeignet oder validiert wären, eine sich ausbildende Peritonitis vorherzusagen [24]. Dabei scheinen frühe postoperative klinische Parameter am ehesten geeignet zu sein, eine beginnende Sepsis vorherzusagen [25]. Insofern ist es notwendig, in der direkten postoperativen
Phase eines Patienten ein intensives Monitoring durchzuführen, um über eine notwendige Relaparotomie in täglicher Abfolge erneut entscheiden zu können.

Untersuchungen $\mathrm{zu}$ spezifischen immunologischen Biomarkern, die eine abdominelle Sepsis vorhersagen können, bleiben jedoch sporadisch. In einer jüngst dazu veröffentlichen Metaanalyse wurde die prädiktive Wertigkeit des C-reaktiven Proteins (CRP) für das frühzeitige Er- kennen infektiöser Komplikationen nach abdominellen Major-Operationen beleuchtet. Die Autoren schlussfolgerten, dass infektiologische Komplikationen in diesen Patienten bei CRP-Werten unter $159 \mathrm{mg} / \mathrm{l}$ am 3. postoperativen Tag sehr unwahrscheinlich waren [26]. Eine weitere Studie zu Patienten im Zusammenhang mit Anastomoseninsuffizienzen kommt zu ähnlichen Ergebnissen. Hier zeigten sich negative prädiktive Werte am 3., 4. und 5. postoperativen Tag, bei insgesamt schwacher positiver prädiktiver Wertigkeit des CRP [27]. Auch eine weitere Auswertung des CRP und der Leukozytenzahlen bestätigte die geringe Wertigkeit in der Vorhersage von Anastomoseninsuffizienzen, speziell nach laparoskopischen kolorektalen Resektionen [28].

\section{I) Interleukin-6 scheint ein vielversprechender Biomarker zu sein}

Dagegen scheint das Interleukin(IL)-6 ein vielversprechender Biomarker für das frühzeitige Erkennen postoperativer Komplikationen, speziell auch der Sepsis, nach elektiven abdominellen Major-Operationen zu sein. So vermag IL-6 bereits am 1. postoperativen Tag Patienten mit infektiologischen Problemen zu diskriminieren, wo hingegen dieses mit dem CRP erst am 3. postoperativen Tag gelang [29]. Ein weiterer möglicher Marker könnte das präoperativ gemessene intestinale fettsäurenbindende Protein sein, im Sinne seiner additiven Wertigkeit in der Risikovorhersage postoperativer Anastomoseninsuffizienzen.

In der Kombination wiesen CRP und Calprotectin nach einer jüngsten Untersuchung aus dem Vorjahr eine hohe diagnostische Genauigkeit in der Vorhersage auftretender Anastomoseninsuffizienzen auf [30]. Bedauerlicherweise sind aus diesen Untersuchungen keine Ergebnisse zur zusätzlichen Wertigkeit der Marker in der Selektion einer frisch einsetzenden Peritonitis bei oftmals bereits septischen Patienten beschrieben. Vor einer Implementierung dieser Marker zur Überwachung von Patienten mit drohender abdomineller Sepsis be- 
darf es somit ihrer weitergehenden Untersuchung.

Die oftmals unsichere Wertigkeit der CT in ihrer Aussage einer sich ausbreitenden abdominellen Infektion im frühen postoperativen Verlauf stellt ein weiteres Problem bei der Überwachung von Patienten und zu treffender Entscheidung zur Relaparotomie dar. So betrug der positive prädiktive Wert der CT in der Erkennung einer abdominellen Sepsis nach elektiver abdomineller Operation 0,71 (95\%-CI: 0,57-0,83) und wies damit eine deutliche Unsicherheit in der Trennschärfe auf. Der negative prädiktive Wert erwies sich mit 0,15 wiederum relativ vertrauenswürdig (95\%-CI: 0,06-0,32; [31]). In der RELAP-Studie wurde lediglich in $18 \%$ der Patienten, in denen ROD erfolgten, eine CT-Untersuchung während der ersten Woche nach initialer Laparotomie durchgeführt und das, obwohl die meisten Relaparotomien in dieser Zeit erfolgten.

Der Einsatz der CT in der Selektion der Patienten für eine Relaparotomie und die daraus gewonnenen Erkenntnisse aus der Interpretation der CT-Untersuchungen werden sehr wahrscheinlich die Effektivität der ROD-Strategie steigern [25, 31]. Dazu wurde in der eigenen Arbeitsgruppe ein Entscheidungsprogramm entwickelt, mit dem die Wahrscheinlichkeit einer sich ausbildenden Sepsis aus einer abdominellen Infektion heraus in Patienten mit operationsbedürftiger sekundärer Peritonitis besser vorhergesagt werden kann (• Abb. 4). Diese Entscheidungshilfe basiert auf frühen postoperativen prädiktiven Faktoren und kann alle 12$24 \mathrm{~h}$ verwendet werden. Basierend auf der erhobenen Risikokategorie sollte eine CT oder eine kurzfristig neu durchzuführende Risikoabschätzung anhand des Prädiktionsmodells bei dem Patienten zur Festlegung des weiteren Prozedere erfolgen [25]. Dieses Entscheidungsinstrument wurde zurückliegend extern validiert (persönliche Mitteilung). Bei insgesamt 69 untersuchten Patienten wurden dazu 161 Risikoabschätzungen unter Einsatz dieses Instruments durchgeführt. Die Diskriminierungskapazität dieses Entscheidungsinstruments erschien angemessen („area under the receiving operator curve“ 0,79 ), wobei die
Auftretenshäufigkeit einer frühen Sepsis signifikant zwischen drei Auswertungskategorien differierte. Ein negativer prädiktiver Wert aus dem Entscheidungsinstrument von $89 \%$ wurde als niedrig kategorisiert. Für die klinische Praxis kann ein negativer prädiktiver Wert in der postoperativen Phase somit eine Hilfe für weitergehende Entscheidungen bedeuten.

\section{Optimierung der interdisziplinären Behandlung}

Die Behandlung der sekundären Peritonitis erfordert einen multidisziplinären Ansatz und notwendigerweise eine enge Zusammenarbeit der Fächer Chirurgie mit Intensivmedizin, diagnostischer Radiologie und Mikrobiologie. Etwa $40 \%$ der Patienten mit sekundärer Peritonitis benötigen im Verlauf ihrer Erkrankung intensivmedizinische Behandlungsmaßnahmen. Bis heute bereitet es immer wieder Besorgnis hinsichtlich des Einflusses der mannigfaltigen intensivmedizinischen Variablen auf die Mortalität und Morbidität des Patienten. Holländische Studien zeigten hierzu den günstigen Einfluss eines hohen Behandlungsaufkommens auf eine insgesamt reduzierte Gesamtmortalität bei den Patienten mit schwerer Sepsis [32]. Die Zentrenbildung für Patienten mit sekundärer Peritonitis erscheint unter Berücksichtigung ihrer hohen Inzidenz weder praktikabel noch möglich. Dennoch sollte die Aufnahme bzw. Verlegung kritisch kranker Patienten in ein Zentrum mit hohem intensivmedizinischem Versorgungsstandard für diese komplexe Erkrankungsform und 24-stündiger sowie 7-tägiger Verfügbarkeit ausgebildeter Intensivmediziner einschließlich interventionell tätiger Radiologen erwogen werden.

\section{Fazit}

- Die Behandlung der sekundären Peritonitis beinhaltet mannigfaltige Aspekte. Die Verbesserung nur eines der Problempunkte wird folglich nicht zu einer nachhaltigen Absenkung der Mortalität und Morbidität bei den betroffenen Patienten führen. Sowohl der grundsätzlich multidisziplinäre Behandlungsansatz als auch die Diagnostik und eng damit einhergehend die Entscheidungsprozesse bedürfen zukünftig weiterer Verbesserung.

- Beispiele für bislang erzielte wichtige Fortschritte in der Behandlung der Peritonitis sind die präventive antifungale Therapie bei Hochrisikopatienten, die berechtigten Zweifel an den Vorteilen einer programmierten abdominellen Lavage und die Erkenntnis zum bedeutungsvollen Bauchdeckenverschluss und der Relaparotomie unter On-demand-Strategie bei allen Patienten unabhängig von der Schwere des Krankheitsbildes.

- Hervorzuheben bleibt die Bedeutung einer engmaschigen Überwachung (Monitoring) des Patienten mit Peritonitis, eines intensiven Einsatzes diagnostischer Methoden und eines über $24 \mathrm{~h}$ sowie 7 Tage vorgehaltenen Entscheidungsfindungsprozesses.

\section{Korrespondenzadresse}

Prof. Dr. M.A. Boermeester

Department of Surgery,

Academic Medical Center

PO Box 22660,1100 DD Amsterdam

m.a.boermeester@amc.uva.nl

\section{Einhaltung ethischer Richtlinien}

Interessenkonflikt. O. Ruler und M.A. Boermeester geben an, dass kein Interessenkonflikt besteht.

Dieser Beitrag beinhaltet keine Studien an Menschen oder Tieren.

Open Access. Dieser Artikel unterliegt den Bedingungen der Creative Commons Attribution License. Dadurch sind die Nutzung, Verteilung und Reproduktion erlaubt, sofern der/die Originalautor/en und die Quelle angegeben sind.

\section{Literatur}

1. Wittmann DH, Schein M et al (1996) Management of secondary peritonitis. Ann Surg 224(1):10-18

2. Lamme B, Boermeester MA et al (2004) Mortality and morbidity of planned relaparotomy versus relaparotomy on demand for secondary peritonitis. Br J Surg 91(8):1046-1054

3. Angus DC, van der Poll T (2013) Severe sepsis and septic shock. N Engl J Med 369(21):2063 
4. van Ruler O, Mahler CW et al (2007) Comparison of on-demand vs planned relaparotomy strategy in patients with severe peritonitis: a randomized trial. JAMA 298(8):865-872

5. Schein M (2002) Surgical management of intraabdominal infection: is there any evidence? Langenbecks Arch Surg 387(1):1-7

6. Boermeester MA (2007) Surgical approaches to peritonitis. Br J Surg 94(11):1317-1318

7. van Westreenen M, Mul FJ et al (1999) Influence of peroperative lavage solutions on peritoneal defence mechanisms in vitro. Eur J Surg 165(11):1066-1071

8. Dellinger RP, Levy MM et al (2013) Surviving Sepsis Campaign: international guidelines for management of severe sepsis and septic shock, 2012. Intensive Care Med 39(2):165-228

9. Barie PS, Hydo LJ et al (2005) Influence of antibiotic therapy on mortality of critical surgical illness caused or complicated by infection. Surg Infect (Larchmt) 6(1):41-54

10. Ibrahim EH, Sherman G et al (2000) The influence of inadequate antimicrobial treatment of bloodstream infections on patient outcomes in the ICU setting. Chest 118(1):146-155

11. Wong PF, Gilliam AD et al (2005) Antibiotic regimens for secondary peritonitis of gastrointestinal origin in adults. Cochrane Database Syst Rev 2:CD004539

12. van Ruler O, Kiewiet JJ et al (2012) Initial microbial spectrum in severe secondary peritonitis and relevance for treatment. Eur J Clin Microbiol Infect Dis 31(5):671-682

13. Hedderwick SA, Lyons MJ et al (2000) Epidemiology of yeast colonization in the intensive care unit. Eur J Clin Microbiol Infect Dis 19(9):663-670

14. van Till JW, van Ruler $O$ et al (2007) Singledrug therapy or selective decontamination of the digestive tract as antifungal prophylaxis in critically ill patients: a systematic review. Crit Care 11(6):R126

15. Blot SI, Vandewoude KH et al (2007) Candida peritonitis. Curr Opin Crit Care 13(2):195-199

16. Kiewiet JJ, van Ruler $O$ et al (2009) Treatment of secondary peritonitis: slow progress. Ned Tijdschr Geneeskd 153:A386

17. Weber DG, Bendinelli C, Balogh ZJ (2014) Damage control surgery for abdominal emergencies. $\mathrm{Br}$ J Surg 101(1):e109-118

18. Burlew CC, Moore EE et al (2011) Sew it up! A Western Trauma Association multi- institutional study of enteric injury management in the postinjury open abdomen. J Trauma 70(2):273277

19. Robledo FA, Luque-de-Leon E et al (2007) Open versus closed management of the abdomen in the surgical treatment of severe secondary peritonitis: a randomized clinical trial. Surg Infect (Larchmt) 8(1):63-72

20. Atema JJ, Gans SL, Boermeester MA (2015) Systematic review and meta-analysis of the open abdomen and temporary abdominal closure techniques in non-trauma patients. World J Surg 39(4):912-925

21. Penninckx FM, Kerremans RP, Lauwers PM (1983) Planned relaparotomies in the surgical treatment of severe generalized peritonitis from intestinal origin. World J Surg 7(6):762-766

22. Zugel N, Siebeck S et al (2002) Circulating mediators and organ function in patients undergoing planned relaparotomy vs conventional surgical therapy in severe secondary peritonitis. Arch Surg 137(5):590-599
23. Opmeer BC, Boer KR et al (2010) Costs of relaparotomy on-demand versus planned relaparotomy in patients with severe peritonitis: an economic evaluation within a randomized controlled trial. Crit Care 14(3):R97

24. van Ruler O, Kiewiet JJ et al (2011) Failure of available scoring systems to predict ongoing infection in patients with abdominal sepsis after their initial emergency laparotomy. BMC Surg 11:38

25. Kiewiet JJ, van Ruler O, Boermeester MA, Reitsma JB (2013) A decision rule to aid selection of patients with abdominal sepsis requiring a relaparotomy. BMC Surg 13:28

26. Gans SL, Atema JJ, van DS, Groot KB, Boermeester MA (2015) Diagnostic value of C-reactive protein to rule out infectious complications after major abdominal surgery: a systematic review and metaanalysis. Int J Colorectal Dis 30(7):861-873

27. Singh PP, Zengh IS et al (2014) Systematic review and meta-analysis of use of serum C- reactive protein levels to predict anastomotic leak after colorectal surgery. Br J Surg 101(4):339-346

28. Pedersen T, Roikjær O, Jess P (2012) Increased levels of C-reactive protein and leukocyte count are poor predictors of anastomotic leakage following laparoscopic colorectal resection. Dan Med J 59(12):A4552

29. Rettig TC, Verwijmeren L et al (2015) Postoperative Interleukin-6 level and early detection of complications after elective major abdominal surgery. Ann Surg (e-pub ahead of print)

30. Reisinger KW, Poeze M et al (2014) Accurate prediction of anastomotic leakage after colorectal surgery using plasma markers for intestinal damage and inflammation. J Am Coll 219(4):744751

31. Go HL, Baarslag HJ, Vermeulen H, Laméris JS, Legemate DA (2005) A comparative study to validate the use of ultrasonography and computed tomography in patients with post- operative intraabdominal sepsis. Eur J Radiol 54(3):383-38

32. Damiani E, Donati A et al (2015) Effect of performance improvement programs on compliance with sepsis bundles and mortality: a systematic review and meta-analysis of observational studies. PLoS One 10(5):e0125827

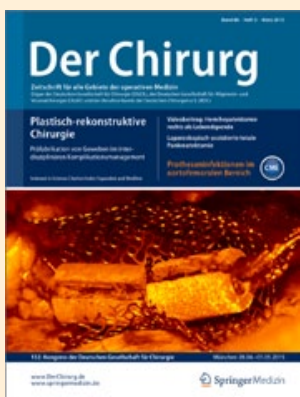

\section{4. „Julius-Springer-Preis für Chirurgie" gestiftet von SpringerMedizin}

Der 2012 ins Leben gerufene „JuliusSpringer-Preis für Chirurgie" wird auch im kommenden Jahr von Springer Medizin verliehen. Der mit 3000 EUR dotierte Preis soll die wissenschaftliche Forschung auf dem Gebiet der Chirurgie unterstützen und insbesondere den Nachwuchs fördern. Prämiert wird die beste frei eingereichte Arbeit der Rubriken „Originalien“ oder „Übersichten”, die im Jahr 2015 in Der Chirurg publiziert wurde. Beurteilt werden die Beiträge durch die Schriftleitung und das Herausgeberboard von Der Chirurg. Als Anerkennung und Wertschätzung der Leistungen unserer Autoren wird zudem im Rahmen eines festlichen Abends im Zuge des DGCH-Kongresses 2016 der Leserpreis für die jeweils herausragendste Arbeit der Rubriken „Leitthema“ und „CME Zertifizierte Fortbildung" vergeben.

\section{Möchten auch Sie eine „Übersicht“ oder "Originalie“ für Der Chirurg ein- reichen?}

Um Ihnen bei der Manuskripterstellung behilflich zu sein, haben wir für unsere Autoren ausführliche Autorenleitfäden und Musterbeiträge zusammengestellt. Diese und weitere Hinweise zur Manuskripterstellung finden Sie auf www.DerChirurg. de

unter dem Menüpunkt „Hinweise für Autoren".

Bitte reichen Sie Ihren fertigen Beitrag online über unser Begutachtungssystem Editorial Manager ein: http://dech.edmgr.com/

Wir freuen uns auf Ihre Beiträge!

\section{Ihre Redaktion Der Chirurg}

